\title{
Dénombrement des spores de Clostridium «butyriques» dans le lait et dans ses contaminants : influence de la présence de lait dans le milieu de culture
}

\section{G. Corrot}

Institut technique de l'élevage bovin, groupement interprofessionnel d'analyses laitières de la HauteMarne, 26, avenue du $109^{e}$ Rl, 52011 Chaumont, France

(reçu le 17 juin 1987, accepté le 5 juin 1989)

Résumé - Pour déterminer si la modification du milieu de culture peut expliquer les discordances observées entre les dénombrements de spores de Clostridium réalisés sur du lait et ceux réalisés sur ses contaminants, nous avons comparé 2 méthodes d'analyse utilisant de l'eau stérile (méthode témoin) ou du lait stérile comme diluant. Nous avons réalisé des dénombrements sur bouses, sur boues de bactofugation et sur des laits ensemencés avec des quantités connues de ces 2 contaminants. Les résultats confirment que la présence de lait dans le milieu entraîne une surestimation des résultats du dénombrement, vraisemblablement par perte de sélectivité du milieu de Bryant et Burkey, en particulier pour les échantillons contenant de la bouse. En revanche, l'utilisation du lait stérile comme diluant permet d'obtenir une meilleure cohérence entre les résultats obtenus sur les contaminants et sur les laits ensemencés avec ceux-ci. Cette méthode peut être recommandée lorsque l'objectif poursuivi est d'analyser la chaîne de contamination en réalisant des dénombrements de spores de "butyriques" sur ensilage, bouse et lait.

spores butyriques — dénombrement - biais - lait -- bouse - milieu de culture

Summary - Butyric acid bacteria spore count in milk and its contaminants : influence of the presence of milk in the culture medium. In order to determine whether the modification of the culture medium explains the differences observed between the counts of Clostridium spores on milk and those evaluated on its contaminants, we compared two methods of analysis using sterile water (control method) or sterile milk as diluent. We carried out counts on cow dung, bactofuge sludge, and on milk inoculated with known quantities of these two contaminants. The results confirm that the presence of milk in the culture medium occasions an overestimation of the counting values, probably owing to the loss of selectivity of the Briant and Burkey medium, especially in samples containing dung. On the other hand, the use of sterile milk as diluent makes it possible to obtain a better coherence between the results achieved in the contaminants and in the milk inoculated with these. This method can thus be recommended when the aim pursued consists of analysing the contamination chain by carrying out counts of butyric acid bacteria spores on silage, cow dung, and milk.

butyric acid bacteria spores - counts - bias - milk - cow dung 


\section{INTRODUCTION}

La réduction à la ferme de la contamination butyrique des laits est d'autant plus efficace qu'il est possible d'établir un diagnostic sûr. Plus précisément, il est important de pouvoir apprécier les risques respectifs liés aux contaminants (ensilage, bouse) et à l'hygiène des animaux et de la traite (Baraton, 1985). Le manque de cohérence fréquemment constaté par les techniciens entre les dénombrements de spores butyriques réalisés sur les différents produits de la chaîne de contamination (ensilage, bouse, lait) limite cette possibilité.

La représentativité des prélèvements ou les conditions de conservation des échantillons peuvent être en cause. Ainsi Baraton (1987) a montré que les dénombrements des spores butyriques dans les bouses étaient 2 fois plus élevés lorsque les échantillons avaient été congelés.

La méthode d'analyse peut aussi jouer un rôle. D'une part, elle ne donne qu'une estimation du nombre le plus probable et il faut tenir compte d'un intervalle de confiance important. D'autre part, lors de l'analyse du lait, l'introduction de celui-ci dans le milieu de culture au lactate provoque une modification de composition par apport de lactose et de protéines. II en résulte une perte de sélectivité du milieu qui risque d'introduire un biais dans les résultats. Cet inconvénient a déjà été mentionné par Bergère et al. (1968). Cette modification n'a pas lieu lors de l'analyse de contaminants du lait, et notamment de bouse.

Le but de cet essai est d'étudier l'importance de ce biais et ses conséquences pratiques pour l'analyse de la chaîne de contamination butyrique.

\section{MATÉRIEL ET MÉTHODES}

\section{Principe de l'étude}

Afin d'éliminer les discordances possibles entre les dénombrements réalisés sur les contaminants et sur le lait, les macérations et les dilutions nécessaires aux analyses ont été effectuées dans les 2 cas avec du lait stérile.

Cette pratique expérimentale a été comparée à la méthode habituelle (témoin) qui consiste à utiliser de l'eau stérile (ou du Ringer) comme milieu de dilution.

Toutes les analyses ont été effectuées par la méthode en milieu de culture liquide au lactate de Bryant \& Burkey modifié par Bergère (CNERNA, 1986).

\section{Schéma expérimental}

Deux essais ont été conduits en utilisant, d'une part la bouse qui constitue le principal contaminant naturel du lait, d'autre part la boue de bactofugation qui est représentative de l'ensemble des contaminations naturelles.

Au total, 8 échantillons (2 contaminants et 6 de laits contaminés) ont été constitués selon le schéma représenté Tableau $\mathrm{I}$.

Chaque échantillon a été analysé avec comme diluant d'une part (méthode expérimentale) du lait stérile, d'autre part (méthode témoin) de l'eau stérile. Chaque analyse a été répétée 15 fois.

\section{Echantillonnage}

L'échantillon de bouse a été constitué à partir de 2 exploitations distribuant une ration à base d'ensilage de maïs. La boue de bactofugation a été fournie par une fromagerie (lait de mélange).

Les 6 laits ont été constitués à partir de lait entier UHT dont l'absence de contamination a été préalablement vérifiée (moins de 20 spores/l). 
Tableau I. Composition des échantillons.

Laits contaminés : Lait stérile $+\left\{\begin{array}{l}\text { Bouse } \\ 1 \mathrm{~g} \text { de contaminant/litre } \\ \text { Lait } 1 \mathrm{~B} \\ 0,1 \mathrm{~g} \text { de contaminant/litre } \\ \text { Lait } 2 \mathrm{~B} \\ 0,01 \mathrm{~g} \text { de contaminant/litre } \\ \text { Lait 3B }\end{array}\right.$

Boue de bactofugation

$34 \mathrm{ml}$ de contaminant/litre Lait $1 \mathrm{bb}$

$6,8 \mathrm{ml}$ de contaminant/litre Lait 2bb

1,36 $\mathrm{ml}$ de contaminant/litre Lait 3 bb

\section{Les analyses}

Les dilutions pratiquées et les nombres de tubes utilisés ont été les suivants :

- bouses : 5 fois 3 tubes à des dilutions décimales de -2 à -6 ;

- boues de bactofugation : 5 fois 3 tubes à des dilutions décimales de -1 à -5 ;

- laits $1: 3$ fois 5 tubes à des dilutions décimales de -1 à -3 ;

- laits 2 et $3: 3$ fois 5 tubes à des dilutions décimales de 0 à -2 .

Les tubes ont été jugés positifs lorsque le bouchon de paraffine était totalement décollé du milieu et le nombre de spores butyriques a été obtenu par la méthode du nombre le plus probable (NPP), en utilisant les tables de De Man (CNERNA, 1986).

\section{Traitement des données}

Tous les calculs ont été effectués sur les logarithmes décimaux des nombres de spores.

Une analyse de variance a été effectuée pour tester l'influence de la méthode d'analyse (expérimentale, témoin), du niveau de contamination $\left(L_{1}, L_{2}, L_{3}\right)$ et l'interaction de ces 2 variables sur le dénombrement des spores.

Les contaminations théoriques des laits, calculées en fonction de la quantité introduite et de la charge en spores butyriques de leurs contaminants, ont été considérées comme non significativement différentes des contaminations réellement observées lorsqu'elles restaient situées à l'intérieur des intervalles de confiance calculés pour un risque de $5 \%$.

\section{RÉSULTATS}

\section{Répétabilité des analyses (Tableau II)}

Les variances des analyses répétées 15 fois sur 16 échantillons (Tableau II) ne sont pas homogènes, avec des extrêmes à 0,025 et à 0,225 (différence hautement significative à $P<0,001$ ).

La répétabilité est moins bonne pour les dénombrements sur les contaminants, qui nécessitent un grand nombre de dilutions successives, que sur les laits. Pour ceuxci, elle est d'autant plus faible que les échantillons sont moins contaminés (Lait 3).

En revanche, on ne note pas de différence sensible de la répétabilité en fonction du type de contaminant ou en fonction du type de diluant. 
Tableau II. Variance moyenne du dénombrement des spores butyriques selon les différents types d'échantillons.

\begin{tabular}{lccc}
\hline Echantillons & $\begin{array}{c}\text { Nombre de séries } \\
\text { d'analyses }\end{array}$ & $\begin{array}{c}\text { Moyenne des } \\
\text { variances }\end{array}$ & $\begin{array}{c}\text { Valeurs extrêmes } \\
\text { des variances }\end{array}$ \\
\hline Laits 1 & 4 & 0,052 & $0,027-0,079$ \\
Laits 2 & 4 & 0,072 & $0,026-0,135$ \\
Laits 3 & 4 & 0,107 & $0,033-0,225$ \\
Contaminants & 4 & 0,135 & $0,087-0,161$ \\
Bouses & 2 & 0,143 & \\
Boues de bactofugat & 2 & 0,125 & $0,027-0,135$ \\
Laits avec bouse & 6 & 0,060 & $0,026-0,225$ \\
Laits avec boue de bactofugat & 6 & 0,095 & $0,026-0,225$ \\
Avec diluant eau & 8 & 0,081 & $0,027-0,161$ \\
Avec diluant lait & 8 & 0,102 & \\
\hline
\end{tabular}

\section{Effets de la méthode d'analyse (Ta- bleaux III et IV)}

\section{Effet sur les contaminants}

En utilisant le lait comme support de dilution, les résultats de dénombrements obtenus pour la bouse et pour la boue de bactofugation sont respectivement 6 et 5 fois plus élevés que ceux obtenus par l'analyse classique (différence hautement significative à $P<0,01$ ).
Effet sur les laits

Pour les laits ensemencés avec de la bouse $\left(L_{1}+L_{2}+L_{3}\right)$, l'effet de la méthode d'analyse sur le résultat du dénombrement est significatif $(P<0,05)$ : en moyenne, le nombre de spores obtenu avec du lait stérile comme support de dilution (19 300 spores/l) est double de celui obtenu par la méthode de référence (10 200 spores/l). En revanche, aucune différence significative n'a été mise en évidence pour les laits ensemencés avec du bactofugat.

Tableau III. Influence de la méthode d'analyse sur les résultats de dénombrement des spores butyriques dans des échantillons de bouses et de laits contaminés avec des bouses (Essai $n^{\circ} 1$ ).

\begin{tabular}{|c|c|c|c|c|}
\hline \multirow{3}{*}{$\begin{array}{l}\text { Type d'échantillon } \\
\text { Bouse (spores/g) } \\
\text { Laits (spores/l) }\end{array}$} & \multicolumn{2}{|c|}{$\begin{array}{l}\text { Méthode expérimentale } \\
\text { (dilutions sur lait) }\end{array}$} & \multicolumn{2}{|c|}{$\begin{array}{l}\text { Méthode témoin } \\
\text { (dilutions sur eau) }\end{array}$} \\
\hline & \multicolumn{2}{|c|}{$\sqrt{ } 120000$} & \multicolumn{2}{|c|}{$\leftarrow 18000$} \\
\hline & $\begin{array}{l}\text { Contamination } \\
\text { théorique }\end{array}$ & $\begin{array}{l}\text { Contamination } \\
\text { observée }\end{array}$ & $\begin{array}{l}\text { Contamination } \\
\text { théorique }\end{array}$ & $\begin{array}{l}\text { Contamination } \\
\text { observée }\end{array}$ \\
\hline LB1 & 120000 & 264200 & 18000 & 58900 \\
\hline LB2 & 12000 & 18930 & 1800 & 11050 \\
\hline LB3 & 1200 & 1440 & 180 & 1630 \\
\hline
\end{tabular}


Tableau IV. Influence de la méthode d'analyse sur les résultats du dénombrement des spores butyriques dans des échantillons de boues de bactofugation de laits contaminés avec ces boues (Essai $\left.n^{\circ} 2\right)$.

Type d'échantillon

Méthode expérimentale (dilutions sur lait)
Méthode témoin

(dilutions sur eau)

\begin{tabular}{|c|c|c|c|c|}
\hline \multicolumn{5}{|c|}{ Bactofugat (spores/l) } \\
\hline Laits (spores/l) & $\begin{array}{c}\text { Contamination } \\
\text { théorique }\end{array}$ & $\begin{array}{c}\text { Contamination } \\
\text { observée }\end{array}$ & $\begin{array}{c}\text { Contamination } \\
\text { théorique }\end{array}$ & $\begin{array}{c}\text { Contamination } \\
\text { observée }\end{array}$ \\
\hline Lbb1 & 22950 & 11900 & 4010 & 11670 \\
\hline Lbb2 & 4590 & 2990 & 800 & 2040 \\
\hline Lbb3 & 920 & 490 & 160 & 490 \\
\hline
\end{tabular}

Interaction du niveau de contamination et de la méthode d'analyse

Elle n'a d'effet significatif sur le résultat du dénombrement que pour les laits contaminés avec de la bouse $(P<0,05)$. L'augmentation des résultats, lorsque le lait est utilisé comme support de dilution, est d'autant plus forte que le niveau de contamina- tion du lait est élevé (Tableau III). Les nombres de spores obtenus sont multipliés par 5 pour LB 1264200 versus 58900 spores/l), par 2 pour $\mathrm{LB}_{2}$ (18 930 versus 11 050), mais sont sensiblement égaux pour LB LB $_{3} 440$ versus 1 630) (Fig. 1).

Pour les laits contaminés avec du bactofugat, aucune interaction n'a été mise en évidence.

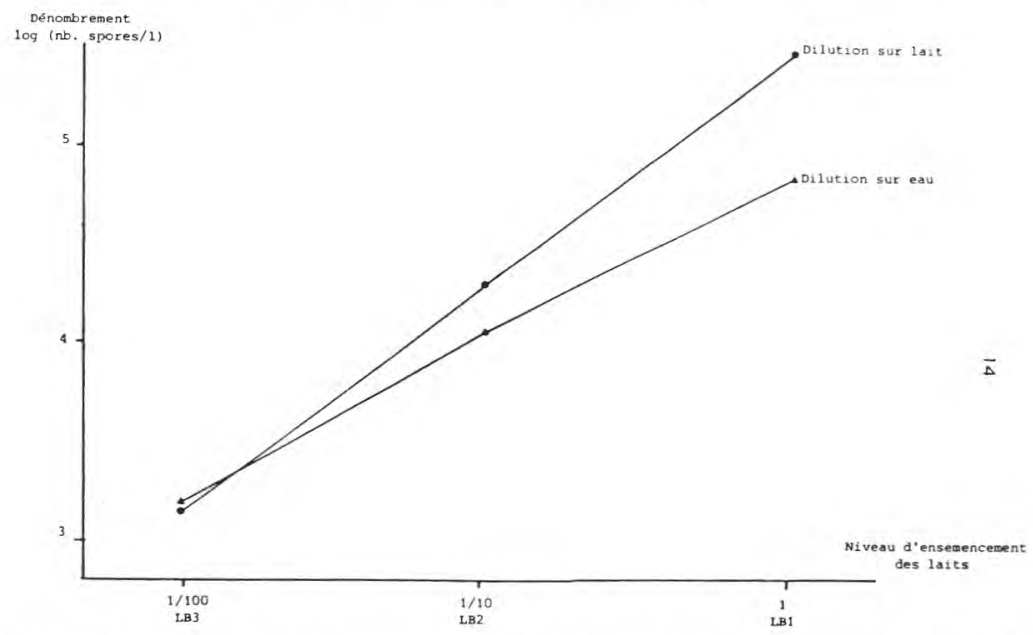

Fig. 1. Interaction du niveau de contamination du lait par des bouses et du milieu de dilution de l'échantillon sur le résultat du dénombrement. 
Comparaison des résultats du dénombrement et de la contamination théorique des laits (Tableaux III et IV)

Quelle que soit la nature du contaminant, les résultats des dénombrements des spores butyriques dans le lait réalisés par la méthode de référence (diluant : eau) sont significativement différents $(P<0,05)$ du nombre théorique, sauf pour le lait $\mathrm{Lbb}_{3}$ qui a la plus grande variance. En revanche, aucune différence significative $(P$ $<0,05)$ entre contamination théorique et observée n'a été mise en évidence lorsque les dénombrements ont été effectués en utilisant du lait stérilisé comme support de dilution.

Enfin, les résultats des dénombrements réalisés en utilisant l'eau comme support de dilution (méthode témoin) ne sont pas significativement différents $(P<0,05)$ des chiffres de contamination théorique des laits calculés à partir du dénombrement obtenu sur les contaminants en utilisant le lait comme support de dilution.

\section{DISCUSSION ET CONCLUSIONS}

Ces résultats montrent que la nature du diluant (lait ou eau) influence les résultats du dénombrement des spores butyriques sur les contaminants et sur les laits contaminés avec de la bouse. On peut penser que la présence de lait entraîne une surestimation des résultats de l'analyse du fait d'une perte de sélectivité du milieu de culture en lactate de Bryant \& Burkey, qui a déjà été signalée par ailleurs (Bergère et al., 1968). Sur le lait contaminé par les bouses, la différence est d'autant plus marquée que le niveau de contamination est plus élevé et nécessite donc un nombre de dilutions, avec du lait ou de l'eau selon le cas, plus important. Le fait qu'aucune différence n'ait été mise en évidence dans le cas des échantillons de lait contaminés avec de la boue de bactofugation peut s'expliquer par les niveaux de contamination plus faibles de ces échantillons. On ne peut exclure toutefois que les différences de comportement vis-à-vis de la méthode d'analyse de ces 2 catégories d'échantillon proviennent de différences dans la population des espèces de Clostridia présentes dans ces 2 produits.

Avec l'analyse habituelle (diluant : eau), les contaminations du lait se trouvent surestimées par rapport à celle de leur contaminant et donc par rapport à leurs contaminations théoriques calculées. Dans ce cas, la surestimation dans la contamination du lait entraîne donc une incohérence avec les résultats des analyses sur les contaminants. Cette surestimation est d'autant plus forte que le lait est moins contaminé, tout au moins dans le cas des bouses. Cela pourrait résulter du fait que pour les faibles contaminations, c'est essentiellement la première série de tubes ensemencée avec des laits non dilués (1 $\mathrm{ml}$ par tube) qui est susceptible de donner des résultats positifs. La contamination d'un lait faiblement contaminé semble donc, dans la pratique, surestimée par rapport à la contamination d'un lait fortement contaminé. Les contaminations plus faibles réalisées avec le bactofugat et le coefficient de dilution plus réduit d'un lait à l'autre peuvent expliquer que ce phénomène ne soit pas apparu dans l'essai $n^{\circ} 2$.

Avec la méthode expérimentale (diluant : lait) au contraire, toutes les contaminations théoriques des laits calculées à partir de celles observées sur les contaminants se trouvent situées à l'intérieur des intervalles de confiance des contaminations réellement obtenues. Lorsque le lait est utilisé comme support de dilution, il n'y a 
donc pas d'incohérence entre les résultats du lait et ceux des produits contaminants, qui sont en fait tous surestimés par rapport à la contamination réelle en Clostridium fermentant le lactate.

Sur le plan pratique, 2 voies apparaissent possibles pour améliorer la cohérence des résultats de dénombrement des spores butyriques sur les contaminants et sur les laits.

On peut tout d'abord envisager d'utiliser la méthode classique, mais en appliquant des corrections systématiques aux résultats des laits. La relation entre la contamination théorique et le résultat de l'analyse (exprimée en unités logarithmiques) semble en effet être linéaire dans l'essai $n^{\circ} 1$ avec la bouse, et l'on pourrait donc envisager de calculer le risque réel, c'est-à-dire la contamination théorique, par régression. A ce propos, une étude (Chamba, 1980) rapporte qu'avec des rations où l'ensilage domine, il existe une bonne corrélation sur les laits d'un groupe d'exploitations entre la contamination en Clostridium tyrobutyricum et la contamination en butyriques totaux (analyse classique). Toutefois, la régression est différente entre le groupe des exploitations distribuant de l'ensilage d'herbe et celui distribuant du maïs. Par ailleurs, la variabilité des résultats ne permet pas d'appliquer avec fiabilité cette correction pour le lait d'une exploitation particulière. Cette voie paraît donc peu praticable.

II paraît donc préférable, pour évaluer le potentiel butyrique d'une exploitation ou dans le cadre d'une étude spécifique de la "chaîne de contamination", d'utiliser du lait stérile comme diluant pour effectuer les dénombrements de spores butyriques en amont du lait (ensilage, bouse), ce qui revient pratiquement à ajouter du lait stérile au milieu de culture à raison de $10 \%(\mathrm{v} / \mathrm{v})$. Cette solution reste toutefois un pis-aller car elle diminue la sélectivité des analyses vis-à-vis des espèces réellement dangereuses qui, dans les bouses par exemple, pourraient être en proportions variables selon le régime alimentaire (Chamba, 1980). On pourrait également envisager de remplacer le lait stérile par un milieu de culture contenant du glucose au lieu de lactate comme le milieu RCM (Hirsch \& Grinsted, 1954), sous réserve d'une vérification préalable de la cohérence des résultats.

On pourrait aussi s'inspirer de la méthode hollandaise de Van Beynum \& Pette (Stadhouders et al., 1985), qui utilise le lait additionné de glucose et acidifié à $\mathrm{pH}$ 5,45 .

L'analyse des laits correspondants pourrait continuer à être effectuée selon la méthode habituelle (milieu de Bryant \& Burkey - eau stérile) sans que cela entrave notablement la cohérence des résultats, puisque les contaminations théoriques des laits déduites des dénombrements réalisés sur les contaminants par la méthode expérimentale restent incluses dans les intervalles de confiance des contaminations observées sur ces laits avec la méthode témoin.

Cette proposition doit être vue comme un palliatif dans l'attente de méthode(s) permettant de dénombrer spécifiquement les spores de Clostridium tyrobutyricum.

\section{REMERCIEMENTS}

Je remercie G. Le Garff (ITEB) et C. Drogeat (ACTA) pour l'aide apportée au dépouillement des résultats, F. Serieys (ITEB) et J.L. Bergère (INRA) pour leurs conseils de rédaction du manuscrit, ainsi que F. Moalic (Laboratoire interprofessionnel de Haute-Marne), grâce auquel ce travail a pu être réalisé. 


\section{RÉFÉRENCES}

Baraton Y. (1985) Limiter la contamination butyrique dans une exploitation. In : Le Point Sur les Butyriques. ITEB, Paris, pp. 21-30

Baraton Y.(1987) Influence du mode de conservation des échantillons sur le résultat du dénombrement des spores butyriques. (Compte rendu d'étude non publié)

Bergère J.L., Gouet P., Hermier J. \& Mocquot G. (1968) Les Clostridium du groupe butyrique dans les produits laitiers. Ann. Inst. Pasteur 19, 41-54

Chamba F. (1980) Comparaison de la contamination butyrique observée dans les fourrages, les bouses et le lait avec différents régimes. In : Compte Rendu d'Éssai ITG-ITEB. ITG, S80/3/ A, Bourg

CNERNA (Commission Qualité Bactériologique) (1986) Recommandations pour l'estimation de la contamination du lait en spores de Clostridia. Rev. Lait Fr. 451, 39-45

Hirsch A. \& Grinsted E. (1954) Methods for the growth enumeration of anaerobic spores. Formers from cheese with observations on the effect of nisin. J. Dairy Res. 21, 101-110

Stadhouders J., Hup G. \& Nieuwenhof F.F. (1985) Silage and cheese quality. Nizo Mededeling $M 19$ A, 1-40 